\title{
AFRONTAMIENTO Y RESILIENCIA: UN ESTUDIO CON ADOLESCENTES SANOS
}

\author{
M. Consuelo Morán-Astorga \\ Universidad de León. León. España \\ mcmora@unileon.es \\ Ester Menezes dos Anjos \\ Faculdade Martha Falcão/Wyden. Manaos. Brasil \\ anjosesther@gmail.com \\ Maria do Perpétuo Socorro Ramalho Barbosa \\ sukyramalho@yahoo.com.br \\ Centro Universitário do Norte - UNINORTE. Manaos. Brasil
}

Fecha de Recepción: 24 Agosto 2019

Fecha de Admisión: 25 Septiembre 2019

\section{RESUMEN}

El objetivo de este estudio fue conocer la influencia o valor predictivo que tiene el afrontamiento sobre la resiliencia psicológica. Se aplicaron dos cuestionarios de auto-informe a 124 adolescentes españoles para medir las estrategias de afrontamiento y la resiliencia. Los datos obtenidos se analizaron con el IBM-SPSS. Se realizaron análisis descriptivos y de regresión lineal. Los resultados informan que determinadas estrategias de afrontamiento como afrontamiento activo, reevaluación positiva, aceptación del problema y religión se asocian a mayor resiliencia. Otras estrategias como autoinculpación, desconexión y uso de sustancias se asocian a más bajas puntuaciones en resiliencia. Los adolescentes estudiados utilizan con mayor frecuencia el afrontamiento activo a la hora de resolver los problemas que les causan estrés, también la planificación y el apoyo emocional. Autodistracción también aparece con alta puntuación. El estudio de la resiliencia y el afrontamiento es un asunto de gran interés científico en el campo de la psicología, fundamentalmente porque en la etapa adolescente vemos conveniente el aprendizaje de las estrategias de afrontamiento que suponen una mayor resiliencia ante el estrés de la vida.

Palabras clave: resiliencia; afrontamiento; psicología positiva; adolescentes.

\section{ABSTRACT}

Coping and resilience in adolescents. The aim of this work was to know the effect value of coping in psychological resilience. Two self-report questionnaires were applied to 124 Spanish adolescents to measure coping strategies and resilience. The data obtained were analyzed with the IBMSPSS. Descriptive and linear-regression analyzes were performed. The results inform that certain 
coping strategies such as Active coping, Positive reframing, Acceptance of the problem and religion are associated with a greater resilience. Other strategies such as Self-Blame, Behavioral Disengagement and Substance Use are associated with lower resilience scores. The adolescents studied use Active Coping more frequently when they solving problems with stress, also Planning and Using Emotional Support. Self-distraction also appears with high score. The study of resilience and coping has a great scientific interest in the field of psychology and health, mainly because in the adolescent stage we find it convenient to learn coping strategies that imply greater resilience to the stress of life.

Keywords: resilience; coping; positive psychology; adolescence.

\section{INTRODUCCIÓN}

La adolescencia es un periodo vital fuente de cambios fisiológicos y psicológicos que en determinadas personas pueden causar estrés (Holmes y Rahe, 1967). Es una etapa que viene acompañada por la llegada de nuevas responsabilidades y de nuevos aprendizajes en el terreno para las relaciones interpersonales. Durante esta etapa, los adolescentes desarrollan un alto sentido de su identidad y adquieren un alto grado de autonomía (Cangas, Fínez, Morán, Navarro y Moldes, 2019).

Son numerosas las críticas que se vierten sobre la actual educación de niños y adolescentes. Estas expresan que a las nuevas generaciones se les proporciona tal grado de confort y de protección que llega a producir un efecto negativo sobre su resiliencia al estrés derivado de las demandas e esta crucial etapa del desarrollo humano.

Por otra parte, la vida de los adolescentes en la actualidad tampoco parece fácil. La seguridad de un entorno familiar estable y sin conflictos explícitos no está garantizada. Las familias actuales tienen pocos miembros, pocos hermanos. Esto produce que los adolescentes carezcan de la protección que brindan los familiares, que antaño proporcionaban ayuda en momentos de conflicto con otros pares y suponían un apoyo en momentos de soledad. El estar solo en este momento de la vida tan crucial como es la adolescencia es un factor de riesgo en sí mismo.

El grupo de hermanos es un grupo social que desempeña casi todas las funciones del grupo humano: proporciona información importante sobre la realidad que te circunda, ayuda en momentos bajos, protege a sus miembros de los ataques, proporciona juegos y entretenimiento en momentos de ocio...

El apoyo social es fundamental para afrontar los retos y desafíos que nos presenta la vida. Vivir significa ir salvando dificultades, resistir y llegar hasta el final siendo eficientes y felices. La mayoría de los problemas que nos encontramos son sencillos, otros son más difíciles, pero todos nos hace ir aprendiendo para afrontar futuros retos y resolverlos de manera exitosa.

Acontecimientos peligrosos también pueden aparecer en algún momento de nuestra vida, con un fuerte potencial traumático (Bonanno y Mancini, 2008). Las diferencias individuales van a determinar la manera de reaccionar a estos eventos (Fletcher y Sarkar, 2013) y más aún cuando el trauma puede ser el resultado.

Es la resiliencia el conjunto de cualidades personales que nos hacen conservar un ajuste ante la adversidad, mantener un equilibrio adaptativo, crecer y desarrollarnos más vigorosamente una vez superado el trauma (Morán et al., 2019).

La resiliencia es vista, más que como la simple recuperación de un trauma, como un desarrollo positivo vigoroso y adaptativo a los siguientes periodos de perturbación homeostática. Aunque se pensó que caracteriza a individuos extraordinarios, investigaciones más recientes sobre la adaptación positiva en respuesta a la adversidad extrema proponen que la resiliencia es relativamente más común entre niños y adolescentes que han estado expuestos a la adversidad, al trauma y al infortunio (Morán, 2013). En adultos, la resiliencia podría definirse como la habilidad para mantenerse rela- 
tivamente sano y con niveles estables de funcionamiento físico y psicológico después de experiencias traumáticas (Luthar y Cicchetti, 2000).

En esta misma línea, Manciaux, Vanistendael, Lecomte y Cyrulnik (2001) la definen como la capacidad de las personas para seguir proyectándose en el futuro a pesar de acontecimientos desestabilizadores, de condiciones de vida difíciles y de traumas a veces graves.

La resiliencia, para Cyrulnik (2001), es la capacidad para mantener una adaptación de las funciones físicas y psicológicas en situaciones críticas, y esto no es una condición absoluta ni se adquiere de una vez para siempre: es el resultado de un proceso dinámico y evolutivo que varía según las circunstancias personales, la naturaleza del trauma, el contexto y la etapa de la vida. Como todo proceso es un devenir, no significa tanto que una persona es resiliente como que lo es su evolución y el proceso de vertebración de su propia historia vital.

¿Pero cuáles son los factores que pueden ser determinantes de la mayor resiliencia? La psicología positiva ha propuesto que algunas características de personalidad como la seguridad en uno mismo y en la propia capacidad de afrontamiento, tener un propósito significativo en la vida, creer que uno puede influir en lo que le sucede y creer que se puede aprender tanto de las experiencias positivas como de las negativas, favorecerían respuestas resilientes. También Fínez y Morán (2014) han hallado que tener un buen autoconcepto y una sana autoestima, o lo que es lo mismo, tener un sesgo positivo en la percepción de uno mismo promueve un mejor ajuste ante la adversidad.

Como precursor de la nueva corriente positiva de la psicología, Seligman (2003) ha encontrado que determinadas fortalezas humanas actúan como amortiguadores contra los trastornos mentales y el trauma. Este investigador también ha hallado suficiente evidencia empírica de que determinadas características positivas como el optimismo, la esperanza, la perseverancia o el valor actúan como barreras contra los trastornos mentales (en Morán, Vicente, Sánchez y Montes, 2014). Este modelo que defiende la psicología positiva de personalidad resiliente refuerza la idea de que las personas auténticas controlan mejor su vida.

Además, existe un acuerdo entre los investigadores en considerar que la manera de afrontar los problemas que en la vida nos causan estrés puede ser uno de los determinantes de la resiliencia (Becoña, 2006). De hecho, Connor y Davidson (2003) definen la resiliencia como una medida de la capacidad que tienen los organismos vivos para enfrentarse con el estrés.

El afrontamiento es cualquier esfuerzo para manejar el estrés, son las cosas que las personas hacen para evitar ser dañadas por las adversidades de la vida. Zeidner y Hammer (1990) definen el afrontamiento "como características o conductas en curso que capacitan a los individuos para manejar los estresores de forma más efectiva, experimentar pocos síntomas o que éstos sean menos severos una vez expuestos al estresor, o recuperarse más rápidamente de la exposición" ( $p$. 694).

Otra característica del afrontamiento es que es una respuesta susceptible de ser aprendida frente a las situaciones problemáticas. También que el afrontamiento requiere un esfuerzo, unas veces consciente, otras no, pero requiere un esfuerzo dirigido a manejar la situación que, en ocasiones, cuando no se puede cambiar, requiere un esfuerzo para adaptarse a ella (Morán, 2009).

Como quiera que estos esfuerzos son diversos, les denominamos estrategias de afrontamiento, definidas como las acciones que los individuos realizan para frenar, amortiguar y, si es posible, anular los efectos de las situaciones amenazantes. Tomando como referencia teórica los trabajos de Lazarus, otros autores (Carver, Scheier y Weintraub, 1989) han desarrollado un modelo multidimensional del afrontamiento. Estos últimos autores crearon un cuestionario denominado COPE para evaluar el afrontamiento y encontraron una serie de estrategias, conceptualmente diferentes, que la mayoría de las personas utilizamos para hacer frente a los problemas que nos causan estrés. 
Posteriormente Carver (1997) realiza una versión breve de ese cuestionario con solo 28 ítems: el Brief COPE. Este cuestionario, con dos ítems por escala, evalúa 14 estrategias de afrontamiento.

\section{OBJETIVOS DE LA INVESTIGACIÓN}

En este trabajo nos planteamos como objetivo averiguar qué estrategias de afrontamiento predicen una mayor resiliencia para superar con éxito las situaciones difíciles de la vida.

\section{MUESTRA Y/O PARTICIPANTES}

Fueron 124 adolescentes entre 16 y 19 años quienes completaron los cuestionarios de autoinforme. La media de edad fue de 16 años y 9 meses; el 57\% eran mujeres. Un investigador experto realizó la aplicación de los instrumentos en un instituto de bachillerato de León ciudad, durante el horario escolar. Los grupos tenían menos de 30 alumnos. Fueron aplicados en un mismo día (primavera de 2018).

\section{METODOLOGÍA Y/O INSTRUMENTOS UTILIZADOS}

CD-RISC 25: es la Connor-Davidson Escala de Resiliencia-25 (Connor y Davidson, 2015) que sirve para evaluar la capacidad de resiliencia psicológica. Es un cuestionario de 25 ítems, se responde en una escala tipo Likert con 5 opciones de respuesta, de 0 a 4 , donde 0 es "nunca" y 4 "significa casi siempre" con puntuaciones intermedias. Es una escala unidimensional, eso significa que da una sola medida, que es la capacidad de resiliencia. El rango de puntuaciones totales de esta escala va de 0 a 100: puntuaciones más altas indican mayor resiliencia.

COPE 28 (Morán, 2019): es un cuestionario de autoevaluación de 28 ítems que se responden en una escala tipo Likert de 0 a 3 puntos (0 significa "no, en absoluto" y 3 , "mucho", con puntuaciones intermedias). El COPE-28 es la versión española del Brief COPE de Carver (1997). Con él se evalúan las siguientes 14 estrategias de afrontamiento: Afrontamiento Activo, Planificación, Apoyo Emocional, Apoyo Social, Religión, Reevaluación Positiva, Aceptación, Negación, Humor y Auto-distracción, Desahogo, Desconexión Conductual, Uso de Sustancias y Autoinculpación. La puntuación máxima que se puede obtener en cada estrategia son 6 puntos, la mínima 0.

\section{RESULTADOS ALCANZADOS}

Tabla 1. Estadísticos descriptivos de las variables estudiadas

\begin{tabular}{l|c|c|} 
& Media & D.T. \\
\hline CD-RISC 25 (puntuación total) & 67,55 & 10,971 \\
\hline Afrontamiento Activo & 4,44 & 1,030 \\
\hline Planificación & 3,77 & 1,274 \\
\hline Apoyo Emocional & 3,87 & 1,459 \\
\hline Apoyo Social & 3,38 & 1,317 \\
\hline Religión &, 74 & 1,331 \\
\hline Reevaluación Positiva & 3,48 & 1,543 \\
\hline Aceptación & 4,29 & 1,354 \\
\hline Negación &, 83 & 1,286 \\
\hline Humor & 2,90 & 2,044 \\
\hline Auto distracción & 3,98 & 1,605 \\
\hline Autoinculpación & 3,02 & 1,677 \\
\hline Desconexión &, 89 & 1,128 \\
\hline Desahogo & 2,61 & 1,627 \\
\hline Uso de sustancias &, 55 & 1,352 \\
\hline N $=124$ & &.. \\
\hline-
\end{tabular}


La tabla 1 nos muestra las puntuaciones medias y la medida de dispersión: la desviación típica (D. T.).

Los adolescentes estudiados utilizan preferentemente estrategias de afrontamiento funcionales como muestran las más altas puntuaciones en afrontamiento activo, planificación y apoyo emocional; aunque también aparece con puntuación alta Auto distracción, que es una estrategia considerada disfuncional.

Tabla 2. Regresión lineal. Resumen del modelo

\begin{tabular}{l|lll|} 
Modelo & $\mathrm{R}$ & $\mathrm{R}^{2}$ & Cambio en $\mathrm{R}^{2}$ \\
\hline 1 &, $436 \mathrm{a}$ &, 190 &, 190 \\
\hline 2 &, $551 \mathrm{~b}$ &, 304 &, 114 \\
\hline 3 &, $607 \mathrm{c}$ &, 368 &, 065 \\
\hline 4 &, $636 \mathrm{~d}$ &, 405 &, 037 \\
\hline 5 &, $653 \mathrm{e}$ &, 427 &, 022 \\
\hline 6 &, $670 \mathrm{f}$ &, 449 &, 022 \\
\hline 7 &, $684 \mathrm{~g}$ &, 468 &, 019 \\
\hline
\end{tabular}
a. Predictores: (Constante), Afrontamiento Activo.
b. Predictores: (Constante), Afrontamiento Activo, Reevaluación Positiva.
c. Predictores: (Constante), Afrontamiento Activo, Reevaluación Positiva, Autoinculpación.
d. Predictores: (Constante), Afrontamiento Activo, Reevaluación Positiva, Autoinculpación, Desconexión.
e. Predictores: (Constante), Afrontamiento Activo, Reevaluación Positiva, Autoinculpación, Desconexión, Aceptación.
f. Predictores: (Constante), Afrontamiento Activo, Reevaluación Positiva, Autoinculpación, Desconexión, Aceptación, Uso de sustancias. g. Predictores: (Constante), Afrontamiento Activo, Reevaluación Positiva, Autoinculpación, Desconexión, Aceptación, Uso de sustancias, Religión.

El análisis de regresión, tabla 2, nos muestra el resumen del modelo. Las estrategias de afrontamiento predicen en un $46,8 \%\left(R^{2}=, 468\right)$ la resiliencia psicológica medida con la CD-RISC.

La variable que entra en primer lugar, con el más alto valor predictivo es afrontamiento activo que predice el 19\% (Cambio en $\mathrm{R}^{2}$ ) de resiliencia. Le sigue reevaluación positiva (11,4\%).

Tabla 3. Coeficientes estandarizados

\begin{tabular}{ll|c|c|c} 
Modelo & Beta & $\mathrm{t}$ & Sig. \\
\cline { 2 - 3 } 7 & (Constante) & & 11,624 &, 000 \\
\hline Afrontamiento Activo &, 135 & 1,678 &, 096 \\
\hline Reevaluación Positiva &, 320 & 4,498 &, 000 \\
\hline Autoinculpación &,- 187 & $-2,553$ &, 012 \\
\hline Desconexión &,- 236 & $-3,040$ &, 003 \\
\hline Aceptación &, 160 & 2,243 &, 027 \\
\hline Uso de sustancias &,- 161 & $-2,291$ &, 024 \\
\hline Religión &, 145 & 2,048 &, 043 \\
\hline
\end{tabular}

a. Variable dependiente: CD-RISC (valor total) 
También entran en el modelo con valores positivos aceptación del problema y religión, con valores más bajos. El valor positivo con el que entra en el modelo predictivo se puede ver en la tabla 3 , tanto en el signo positivo o negativo de Beta 0 de t.

Autoinculpación (6\%), desconexión y uso de sustancias entran en el modelo con un valor negativo; esto significa que influyen en tener menor resiliencia en los adolescentes, como puede verse en la tabla 3 en los valores de $B$ y t.

\section{CONCLUSIONES}

Los adolescentes estudiados utilizan preferentemente estrategias de afrontamiento funcionales como muestran las más altas puntuaciones en Afrontamiento Activo, Planificación y Búsqueda de Apoyo Emocional; aunque también aparece con puntuación alta Auto-distracción que es una estrategia disfuncional.

Las estrategias de afrontamiento predicen en un alto grado la resiliencia psicológica, casi un $50 \%$ de la misma parece estar relacionada con el uso de las estrategias denominadas funcionales. Siendo el Afrontamiento Activo, esto es, concentrando todos los esfuerzos en hacer algo por salir de la situación o intentando por todos los medios que la situación mejore. La siguiente estrategia importante en predecir mayor resiliencia es la Reevaluación Positiva, que significa intentar ver el problema de otra manera, haciendo que parezca más positivo, o también buscar algo bueno en lo que está sucediendo. Con menor valor predictivo entran, también de manera positiva Aceptación del problema y Religión.

Otras estrategias predicen menor resiliencia: son Autoinculpación, Desconexión y Uso de Sustancias que entran en el modelo con un valor negativo.

\section{REFERENCIAS BIBLIOGRÁFICAS}

Becoña, E. (2006). Resiliencia: Definición, características y utilidad del concepto. Revista de Psicopatología Clínica, 11(3), 125-146.

Benavente-Cuesta, M.H. y Quevedo-Aguado M.P. (2018). Resiliencia, bienestar psicológico y afrontamiento en universitarios atendiendo a variables de personalidad y enfermedad. Revista de Psicología y Educación / Journal of Psychology and Education, 13(2), 99-112

Bonanno GA. y Mancini AD. (2008). The human capacity to thrive in the face of potential trauma. Pediatrics. 121(2), 369-75. doi:10.1542/peds.2007-1648.

Cambil-Contreras, M. (2015). Resiliencia, estrategias de afrontamiento y enfoques de aprendizaje, como aspectos de la competencia aprender a aprender con estrés en el contexto de enseñanza aprendizaje universitario (Tesis doctoral). Universidad de Granada (España).

Cangas A.J., Fínez M.J., Morán C., Navarro N., Moldes P. (2019). Mental Health, New Technologies, and Wellbeing for Adolescents. En: Steinebach C., Langer Á. (eds.) Enhancing Resilience in Youth. Cham, Suiza: Springer.

Carver, C. S. (1997). You want to measure coping but your protocol's too long: Consider the Brief COPE. International Journal of Behavioral Medicine, 4(1), 92-100.

Carver, C. S., Scheier, M. F. y Weintraub, J. K. (1989). Assessing coping strategies: A theoretically based approach. Journal of Personality and Social Psychology, 56, 267-283.

Connor, K. M., y Davidson, J. R. T. (2003). Development of a new resilience scale: The ConnorDavidson Resilience Scale (CD-RISC). Depression and Anxiety, 18, 71-82.

Connor, K. M., y Davidson, J. R. T. (2015). Escala de Resiliencia de Connor-Davidson (CD-RISC). Disponible en: https://www.researchgate.net/publication/277139516_Escala_de_ Resiliencia_de_Connor-Davidson_CD-RISC [accessed Apr 04 2019].

Cyrulnik, B. (2001). Los Patitos feos: la resiliencia: una infancia infeliz no determina la vida. Madrid: Gedisa. 
Dos Anjos, E. M. y Morán, M.C. (2016). A personalidade resiliente: Uma conceptualização teórica. International Journal of Developmental and Educational Psychology. Revista INFAD de Psicología, 2(1), 151-156. D0I: http://dx.doi.org/10.17060/ijodaep.2016.n1.v2.297

Fínez, M.J. y Morán, M.C. (2017). Resiliencia y autovaloraciones esenciales: estudio comparativo en adolescentes y jóvenes. Psychology, Society, \& Education, 9(3), 347-356. Doi: $10.25115 /$ psye.v9i3.857.

Fínez, M.J. y Morán, M.C. (2014). Resiliencia y autoconcepto: su relación con el cansancio emocional en adolescentes. International Journal of Developmental and Educational Psychology. Revista INFAD de Psicología, 1(6), 289-296. https://doi.org/10.17060/ijodaep.2014.n1.v6.746.

Fínez, M.J. y Morán, M.C. (2015). La resiliencia y su relación con salud y ansiedad en estudiantes españoles. International Journal of Developmental and Educational Psychology. Revista INFAD de Psicología, 1 (1), 409-416. http://dx.doi.org/10.17060/ijodaep.2015.n1.v1.90.

Fínez, M.J. Morán, M.C., Urchaga, J.D. (2019). Resiliencia psicológica a través de la edad y el sexo. International Journal of Developmental and Educational Psychology. Revista INFAD de Psicología, 1(4), 85-94.

Fletcher, D. y Sarkar, M. (2013). Psychological resilience: A review and critique of definitions, concepts, and theory. European Psychologist, 18(1), 12-23. DOI: 10.1027/1016-9040/a000124

Holmes, T. y Rahe, R. (1967). The social readjustment rating scale. J. Psychoson. Res., 11, 213-218.

Lazarus, R. y Folkman, S. (1984). Estrés y procesos cognitivos. Barcelona: Martínez Roca.

Luthar, S.S. y Cicchetti, D. (2000). Child Dev., 71(3), 543-562.

Manciaux, M., Vanistendael, S., Lecomte, J. y Cyrulnik, B. (2003). La resiliencia: estado de la cuestión. En M. Manciaux (comp.), La resiliencia: resistir y rehacerse. Madrid: Gedisa.

Morán, C. (2019). Estrés, burnout y mobbing. Recursos y estrategias de afrontamiento con instrumentos para su evaluación. León, España: Eolas.

Morán, M.C., Finez, M.J., Anjos, E.M., Pérez-Lancho, M.C., Urchaga, J.D. y Vallejo, G. (2019). Estrategias de afrontamiento que predicen mayor resiliencia. International Journal of Developmental and Educational Psychology. Revista INFAD de Psicología 1(4), 183-190.

Morán, C., Landero, R., y González, M.T. (2010). COPE-28: un análisis psicométrico de la versión en español del Brief COPE. Universitas Psychologica, 9 (2), 543-552.

Morán, M.C. Vicente, F., Sánchez-Cabaco, A. y Montes, E. (2014). Psicología positiva. Antigua 0 nueva concepción. International Journal of Developmental and Educational Psychology, 3, (1), 439-450.

Morán, M.C., Fínez, M. J. y Fernández-Abascal. E. (2017). Sobre la felicidad y su relación con tipos y rasgos de personalidad. Clínica y Salud, 28, 59-63. http://dex.doi.org/:10.1016/j.clysa.2016.11.003

Seligman, MEP. (2003). The past and future of positive psychology. Flourishing: Positive psychology and the life well-lived, 2003, 11-20.

Urchaga-Litago, C Morán-Astorga, MJ Fínez-Silva. 2019. La religiosidad como fortaleza humana. International Journal of Developmental and Educational Psychology INFAD Revista de Psicología, 1(1). 309-316.

Zeidner, Z. y Hammer, AL. (1990). Life events and coping resources as predictors of stress symptoms in adolescents. Personality and Individual Differences, 7,(11), 693-703 
